\title{
The relationship between water vapour saturation of inhaled air and nasal patency
}

\author{
J. Lindemann, R. Leiacker, G. Rettinger, T. Keck
}

\begin{abstract}
The relationship between water vapour saturation of inhaled air and nasal patency. J. Lindemann, R. Leiacker, G. Rettinger, T. Keck. C) ERS Journals Ltd 2003.

ABSTRACT: The nasal cavity volume and the temperature of the nasal mucosa are considered to be the most important predictors of nasal conditioning. The aim of this study was to assess the relationship between the intranasal degree of water vapour saturation of inhaled air and nasal patency.

Intranasal humidity values at different locations within the nasal cavity of $\mathbf{1 5}$ healthy subjects were compared to nasal airway resistance detected by active anterior rhinomanometry (AAR). Repeated measurements were carried out during one day to obtain varying nasal cavity volumes due to the nasal cycle. The end-inspiratory humidity data were obtained with a miniaturised capacitive humidity sensor at defined detection sites within the anterior nasal segment without interruption of nasal breathing. Measurements were carried out at four different times during one day.

The degree of water vapour saturation did not correlate with the values of the AAR at any intranasal detection site and time of detection during one day.

The study supports the view that there is no correlation between the degree of water vapour saturation within the anterior nasal segment and the nasal resistance during the nasal cycle over the day. Although nasal patency varies because of the nasal cycle, the changes of nasal cavity volume due to the nasal cycle do not seem to influence the degree of water vapour saturation of the inspiratory air.
\end{abstract}

Eur Respir J 2003; 21: 313-316.

Dept of Otorhinolaryngology, University of Ulm, Ulm, Germany.

Correspondence: J. Lindemann, Dept of Otorhinolaryngology, University of Ulm, Prittwitzstrasse 43, D-89075 Ulm, Germany.

Fax: 4973150026703

E-mail: joerg.lindemann@medizin.uniulm.de

Keywords: Air condition

nasal cycle

nasal humidity

nasal patency

saturation

Received: July 92002

Accepted after revision: September 30 2002
The blood temperature distribution along the airway walls reflecting the nasal mucosal temperature and the nasal cavity volume are considered to be the two most important parameters of the human air conditioning process as described by HANNA and SCHERER [1] in a theoretical model. Both of these parameters determine the local heat and water exchange in the respiratory system.

The parameter "nasal cavity volume" changes periodically over the day during the so-called nasal cycle. The nasal cycle was first described by KAYSER [2]. Spontaneous changes of unilateral nasal airflow in man are a physiological phenomenon where each side of the nose alternates reciprocally through phases of congestion and decongestion over the day with period lengths ranging from $\sim 1-5 \mathrm{~h}$ [3]. Yet, the understanding of this phenomenon and its description in the literature is still very confusing [4].

The nasal cycle is produced by alterations in autonomic tone of the nasal vasculature and asymmetries in left and right cerebral electroencephalographic activity. The pacemaker for the nasal cycle is believed to be located within the suprachiasmatic nucleus of the hypothalamus [3, 5]. Additionally, a complex interaction of different neurotransmitters (e.g. noradrenaline, acetylcholine, neuropeptide $\mathrm{Y}$, vasoactive intestinal peptide, peptide histidine leucine, substance $\mathrm{P}$ and calcitonin gene-related peptide) seem to be involved [6].
The nasal cycle has been demonstrated using several techniques, including anterior rhinoscopy, rhinomanometry, acoustic rhinometry, rhinostereometry and magnetic resonance imaging [7-9].

The physiological importance and effect of the nasal cycle is still unclear. It does seem to have an effect on mucociliary clearance, nasal inflammatory response and respiratory defence [10-12]. In the literature, the correlation between the objective measurements of nasal airway resistance, including magnetic resonance imaging and subjective assessment of nasal patency is usually regarded as poor $[13,14]$.

Although humidification of air is one of the most important functions of the nose, data about intranasal humidity and nasal airway resistance during the nasal cycle over the day is still missing.

The aim of the present study was to examine a possible relationship between the intranasal degree of saturation within the anterior nasal segment and nasal airway resistance over one day in healthy subjects. For this purpose, repeated measurements of intranasal humidity and airway resistance reflecting changes of the nasal cavity volume due to the nasal cycle were compared.

\section{Material and methods}

The study was performed after receiving approval of the local ethical committee. All experimental 
procedures were explained in full detail to the study participants, who provided written informed consent.

\section{Study subjects}

Altogether, 15 healthy subjects with normal nasal anatomy were included into the study (seven males and eight females) with an average age of $30 \mathrm{yrs}$ (range, 25-42 yrs).

The study participants had no nasal complaints or history of nasal disease and no allergy. All subjects underwent an otorhinolaryngological examination including anterior rhinoscopy and endoscopy of the nasal cavity.

\section{Humidity recording and active anterior rhinomanometry}

For detection of the degree of saturation (relative humidity) of inhaled air a capacitive thin-film humidity sensor (Humichip 17204 HM; Vaisala, Vantaa, Finland) was used. The capacitance of the sensor's polymer film changes sensitively with the absorption of water and is a measure of relative humidity [15].

The humidity sensor was incorporated in an acrylic glass cube and connected to a suction system. Respiratory air was transported to the humidity sensor by means of a silicon suction probe with an outer diameter of $2.5 \mathrm{~mm}$ and an inner diameter of $1.5 \mathrm{~mm}$. The temperature of the interior wall of the silicon suction probe was $37^{\circ} \mathrm{C}$ (body temperature). The authors did not observe a condensation of water because of the short length of the suction probe as demonstrated in several previous investigations. The volume of air sampled through the suction tube came to $17 \mathrm{~mL} \cdot \mathrm{s}^{-1}$. Thus relative humidity between $0-100 \%$ could be measured. In high-velocity air it took $<2 \mathrm{~s}$ to reach $90 \%$ of the steady-state.

All participants had to adapt to the laboratory environment with a room temperature of $25 \pm 1^{\circ} \mathrm{C}$ and relative humidity of $30 \pm 4 \%$ for $30 \mathrm{~min}$ while breathing quietly through the nose in an upright position. Active anterior rhinomanometry (AAR) (Rhinomanometer 300; Atmos, Lenzkirch, Germany; transnasal reference pressure, $150 \mathrm{~Pa}$ ) was performed on both sides.

Humidity recordings were then carried out, placing the suction probe consecutively at the level of the nasal valve area, close to the head of the inferior turbinate and at the level of the anterior turbinate area, close to the head of the middle turbinate in the centre of the air stream, by means of a nasal speculum and a headlight (fig. 1). Nasal endoscopy was then performed in the same nostril in which the sensor was inserted in order to check its correct position. For this reason the nasal endoscope was only temporarily inserted into the nasal entrance before each measurement without touching or irritating the nasal mucosa, as the suction probe was only inserted into the anterior nasal segment. Both nasal cavities were measured one after the other, always starting with the right side. Humidity in the air stream at each site of the nasal cavity was continuously recorded during quiet breathing for $1 \mathrm{~min}$ without interruption of

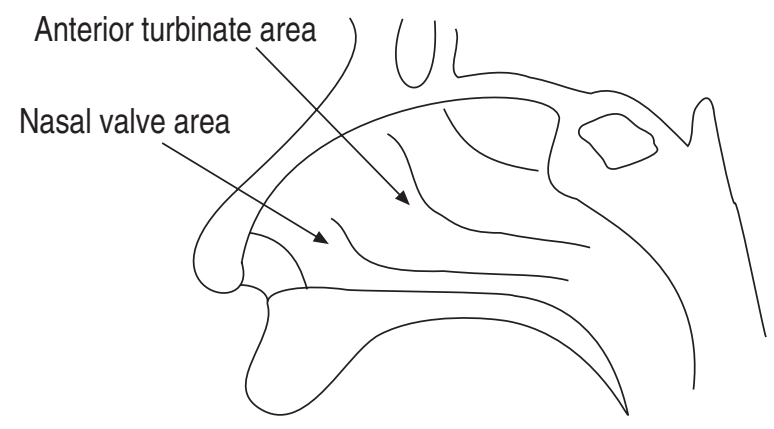

Fig. 1.- Intranasal detection sites (arrows). The suction probe was inserted through the nostril to the desired position in the centre of the air stream under endoscopic control. Both nasal cavities were measured one after the other, always starting with the right side.

physiological nasal breathing. The head of the patient was fixed on a head holder. In all subjects, rhinomanometry and humidity measurements were concurrently performed at 08:00, 11:00, 14:00 and 16:00 h.

\section{Registration of the respiratory cycle}

The phase of inspiration and expiration during the respiratory cycle was continuously detected using a stress sensitive belt around the thorax of each subject (MAP, Martinsried, Germany). The signal of the sensor, integrated in the belt, was amplified and continuously recorded.

\section{Data processing and analysis}

The humidity data were amplified and transferred to a computer via an analogue to digital card. Continuous registration using the computer program Turbo Lab (Bressner Technology, Munich, Germany) was achieved. Respiratory cycles of $1 \mathrm{~min}$ at each detection site were analysed. The mean end-inspiratory humidity of one respiratory cycle was calculated when $\geqslant 5$ repeated inspiration and expiration manoeuvres following each other showed a deviation of endinspiratory temperature values $<3 \%$. When the deviation was higher, measurements were repeated after an interval of $5 \mathrm{~min}$.

\section{Statistical analysis}

The Spearman signed rank test was used to compare humidity results to rhinomanometrical values. The nonparametric Wilcoxon signed rank test was used for comparison of the values of the right and left nasal cavity. The null hypothesis (no difference between the values of the right and left side) was to be rejected at the alpha $=0.05$ significance level.

\section{Results}

There were no individual or technical problems in inserting the suction probe into the nasal cavity. Endoscopic control and mucosal adaptation prior to 
measurements were sufficient to avoid influences like mucosal irritation by the suction probe.

\section{Correlation between end-inspiratory degree of saturation and rhinomanometry}

The median inspiratory flow rates $\left(\mathrm{cm}^{3} \cdot \mathrm{s}^{-1}\right)$ at a transnasal pressure of $150 \mathrm{~Pa}$, of the right side, over the day (4 measurements; 08:00, 11:00, 14:00 and $16: 00 \mathrm{~h}$ ) were 228.0 (range, 20.0-728.0) and of the left side 116.0 (range, 30.0-772.0). The median bilateral inspiratory flow rate was 371.0 (range, 60.0 978.0) (table 1). All of the subjects exhibited a nasal cycle. There was no significant difference in the degree of saturation between the right and the left nasal cavity at both detection sites during the four measurements $(\mathrm{p}>0.05)$.

The median end-inspiratory degree of saturation at the level of the nasal valve area of both sides over the day was 90.1 (range, 52.8-98.0). At the level of the anterior turbinate area the median end-inspiratory degree of saturation of both sides over the day was 89.9 (range, 59.1-98.7.0) (table 1).

There was no statistically significant correlation between the end-inspiratory degree of saturation and nasal airway resistance neither in the nasal valve area $(\mathrm{r}=-0.10)$ nor in the anterior turbinate area $(r=-0.12)$ in all subjects.

\section{Discussion}

The nasal cycle is a physiological fluctuation of nasal patency due to periodic phases of congestion and decongestion of the nasal mucosa on both the right and left nasal conchae over a period of several hours [7]. Around $80 \%$ of the healthy population show a regular cycle [4].

ECCLES [11, 16] reported that the nasal cycle may have a special role in respiratory defence by alternation of air conditioning between the nasal passages, generation of plasma exudates, which physically cleanses the epithelium and provides a source of antibodies and inflammatory mediators, and maintaining the patency of the airway during the inflammatory response to infection. SoANE et al.

Table 1. - Median end-inspiratory degree of water vapour saturation within the nasal valve area (NVA) and the anterior turbinate area (ATA) and the median inspiratory flow rates at a transnasal pressure of $150 \mathrm{~Pa}$ of the right and the left side over the day

\begin{tabular}{|c|c|c|c|c|c|c|}
\hline \multirow[t]{3}{*}{ Time } & \multicolumn{4}{|c|}{ Saturation } & \multicolumn{2}{|c|}{$\begin{array}{l}\text { Flow rates at } \\
150 \mathrm{~Pa} \mathrm{~cm}^{3} \cdot \mathrm{s}^{-1}\end{array}$} \\
\hline & \multicolumn{2}{|c|}{ NVA } & \multicolumn{2}{|c|}{ ATA } & \multirow[t]{2}{*}{ Right } & \multirow[t]{2}{*}{ Left } \\
\hline & Right & Left & Right & Left & & \\
\hline 08:00 & 93.2 & 91.6 & 94.1 & 90.4 & 246 & 138 \\
\hline 11:00 & 89.1 & 88.2 & 88.3 & 85.6 & 231 & 168 \\
\hline 14:00 & 82.4 & 82.7 & 87.0 & 81.9 & 286 & 165 \\
\hline $16: 00$ & 81.8 & 83.8 & 84.9 & 82.3 & 224 & 190 \\
\hline
\end{tabular}

Data are presented as $\%$ unless otherwise stated. $n=15$.
[10] demonstrated that the nasal cycle also has a noticeable effect on the nasal mucociliary clearance.

Although many physiological properties of the nose alternate with the nasal cycle and nasal airway resistance, whether this has any effect on the degree of intranasal saturation of inhaled air with water vapour is less clear. Additionally, the relationship between objective measurements of nasal airway resistance and personal estimation of nasal patency is frequently considered to be poor. Data about intranasal humidity at defined intranasal detection sites and varying nasal airway resistance due to the nasal cycle at different times over the day is still absent.

The nasal cavity volume and the temperature of the nasal mucosa are considered to be the most important predictive factors of nasal conditioning as described by HANNA and SCHERER [1] in a theoretical computer model.

Fluctuations of the nasal airway resistance because of the nasal cycle also reflect changes of the nasal cavity volume. For this reason, rhinomanometrical measurements seem to be suitable for the registration of the effects of the nasal cavity volume on the intranasal degree of saturation. Therefore this study was designed to investigate in vivo the relationship between the degree of intranasal saturation of inhaled air with water vapour and nasal airway resistance.

A miniaturised humidity sensor was used for intranasal measurements of the degree of saturation. The suction probe of the sensor allowed humidity recording at defined intranasal sites in the anterior segment in the centre of the air stream without interruption of nasal breathing. Numerous previous studies showed that the humidity sensor used is suitable for in vivo measurements in the human nasal cavity at defined detection sites [17-19]. Nasal resistance was measured using AAR.

In several investigations, a relative humidity of $\sim 90-100 \%$ in the nasopharynx could be observed [20, 21]. These values are similar to the authors previous observations.

In these studies neither humidity nor temperature measurements in the anterior segment of the nose, nor separation of values at end of inspiration and expiration were performed. RouADI et al. [20] performed measurements in the nasopharynx during nasal breathing through only one nostril because the other nasal cavity was closed by the detection equipment. The particular features of the experimental setup in the current study are that the authors were able to measure relative humidity at defined intranasal detection sites, without interruption of physiological nasal breathing, without blocking one side of the nose and with registration of the respiratory cycle.

The intranasal humidity values depend on the exact intranasal detection site and the time of detection within the respiratory cycle. Several previous measurements of relative humidity at different sites within the nasal cavity showed that in particular, the anterior nasal segment including the nasal valve area seems to be a main area of humidification of inspired air [19]. Therefore, the authors decided to present the humidity values of the anterior nasal segment.

The end-inspiratory degree of saturation was not 
found to correlate with the values of the AAR at any intranasal detection site in the anterior segment and time of detection during the nasal cycle over one day. No comparable data in the literature concerning the influence of the nasal cycle and nasal airway resistance on the intranasal humidity could be found.

There were no significant differences in the values of relative humidity and temperature between the right and the left nasal cavity in previous studies, indicating that the state of mucosal swelling alone is not responsible for air conditioning [17, 18, 22]. In addition, Akerlund and Bende [23] did not find a correlation between the nasal mucosal temperature and nasal airway resistance due to mucosal swelling. Widening of the nasal cavity by mucosal decongestion also did not lead to significant changes of the intranasal humidity profile, temperature profile and particle deposition after topical application of the vasoconstrictor xylometazoline [24, 25].

It is probable that the changes in the airflow pattern (velocity, flow, turbulences) during the nasal cycle lead to different contact times of the inhaled and exhaled air with the surrounding nasal mucosa. Thus, possible changes in the air conditioning and cleansing of the inhaled air due to mucosal congestion and decongestion during the nasal cycle might be compensated.

These results are slightly contradictory to the theoretical hypothesis of HANNA and SCHERER [1]. This contrast may be due to the negligence of changes in the airflow pattern. Further studies are needed and will be performed by the authors working group to investigate the influence of the nasal cavity volume and nasal mucosal temperature on nasal air conditioning. Therefore, in a second step, simultaneous measurements of the nasal mucosal temperature, intranasal airflow humidity and temperature will be performed additionally to AAR, as well as acoustic rhinometry for measurement of the nasal cavity volume.

\section{Conclusion}

The present study supports the view that there is no significant correlation between the degree of water vapour saturation and the nasal resistance during the nasal cycle reflecting changes in the nasal cavity volume over the day. Although nasal patency varies during the nasal cycle, mucosal congestion and decongestion do not seem to influence the end-inspiratory degree of saturation in the anterior nasal segment measurable in the experimental set-up of this study.

\section{References}

1. Hanna LM, Scherer PW. A theoretical model of localized heat and water vapour transport in the human respiratory tract. J Biomec Eng 1986; 108: 19-27.

2. Kayser R. Die exacte Messung der luftdurchgängigkeit der Nase. Arch Rhinol Laryngol 1895; 3: 101-120.

3. Mirza N, Kroger H, Doty RL. Influence of age on the 'nasal cycle'. Laryngoscope 1997; 107: 62-66.

4. Flanagan P, Eccles R. Spontaneous changes of unilateral nasal airflow in man. A re-examination of the 'nasal cycle'. Acta Otolaryngol 1997; 117: 590-595.

5. Galioto G, Mevio E, Galioto P, Fornasari G, Cisternino M,
Fraietta L. Modifications of the nasal cycle in patients with hypothalamic disorders: Kallmann's syndrome. Ann Otol Rhinol Laryngol 1991; 100: 559-562.

6. Lund VJ. Nasal physiology: neurochemical receptors, nasal cycle, and ciliary action. Allergy Asthma Proc 1996; 17: 179-184.

7. Moinuddin R, Mamikoglu B, Barkatullah S, Corey JP. Detection of the nasal cycle. Am J Rhinol 2001; 15: 35-39.

8. Gungor A, Moinuddin R, Nelson RH, Corey JP. Detection of the nasal cycle with acoustic rhinometry: techniques and applications. Otolaryngol Head Neck Surg 1999; 120: 238-247.

9. Fisher EW, Scadding GK, Lund VJ. The role of acoustic rhinometry in studying the nasal cycle. Rhinology 1993; 31: 57-61.

10. Soane RJ, Carney AS, Jones NS, et al. The effect of the nasal cycle on mucociliary clearance. Clin Otolaryngol 2001; 26: 9-15.

11. Eccles RB. The nasal cycle in respiratory defence. Acta Otorhinolaryngol Belg 2000; 54: 281-286.

12. Eccles R, Reilly M, Eccles KS. Changes in the amplitude of the nasal cycle associated with symptoms of acute upper respiratory tract infection. Acta Otolaryngol 1996; 116: 77-81.

13. Passali D, Mezzedimi C, Passali GC, Nuti D, Bellussi L. The role of rhinomanometry, acoustic rhinometry, and mucociliary transport time in the assessment of nasal patency. Ear Nose Throat J 2000; 79: 397-400.

14. Saunders MW, Jones NS, Kabala JE. Parameters of nasal airway anatomy on magnetic resonance imaging correlate poorly with subjective symptoms of nasal patency. Clin Otolaryngol 1999; 24: 431-434.

15. Ohhashi T, Sakaguchi M, Tsuda T. Human perspiration measurement. Physiol Meas 1998; 19: 449-461.

16. Eccles R. A role for the nasal cycle in respiratory defence. Eur Respir J 1996; 9: 371-376.

17. Lindemann J, Kühnemann S, Stehmer V, Leiacker R, Rettinger G, Keck T. Temperature and humidity profile of the anterior nasal airways in patients with nasal septal perforation. Rhinology 2001; 39: 202-206.

18. Lindemann J, Leiacker R, Stehmer V, Rettinger G, Keck T. Intranasal temperature and humidity profile in patients with nasal septal perforation before and after surgical closure. Clin Otolaryngol 2001; 26: 433-437.

19. Keck T, Leiacker R, Heinrich A, Kühnemann S, Rettinger G. Humidity and temperature profile in the nasal cavity. Rhinology 2000; 38: 167-171.

20. Rouadi P, Baroody FM, Abbott D, Naureckas E, Solway J, Naclerio RM. A technique to measure the ability of the human nose to warm and humidify air. J Appl Physiol 1999; 87: 400-406.

21. Cole P. Further observations on the conditioning of respiratory air. J Laryngol 1953; 67: 669-681.

22. Keck T, Leiacker R, Riechelmann H, Rettinger G. Temperature profile in the nasal cavity. Laryngoscope 2000; 110: 651-654.

23. Akerlund A, Bende M. Nasal mucosal temperature and the effect of acute infective rhinitis. Clin Otolaryngol 1989; 14: 529-534.

24. Keck $T$, Leiacker $R$, Schick $M$, Rettinger $G$, Kühnemann S. Temperature and humidity profile of the nasal airways before and after nasal decongestion with xylometazoline. Laryngorhinootologie 2000; 79: 749-752.

25. Keck T, Leiacker R, Kühnemann S, Rettinger G, Lindemann J. Detection of particles within the nasal airways before and after nasal decongestion. Clin Otolaryngol 2001; 26: 324-328. 